\title{
El patrimonio turístico, las actividades económicas vinculadas al ecoturismo y su relación con la vulnerabilidad al cambio climático en los municipios de la Huasteca Potosina
}

\author{
The turistic heritage, economic activities linked to ecotourism \\ and its relation to vulnerability to climate change \\ in the municipalities of the Huasteca Potosina
}

\author{
Francisco Javier Segura Mojica* \\ Tecnológico Nacional de México, México
}

\section{RESUMEN}

En la presente investigación se plantea como objetivo identificar y estimar la relación estadística que existe entre el potencial ecoturístico y las actividades económicas vinculadas al turismo, como es el caso de la oferta de servicios de alojamiento, servicios de alimentos y bebidas, y la vulnerabilidad al cambio climático en los municipios de la zona Huasteca Potosina. Se obtienen los datos correspondientes al índice turístico natural y cultural por municipio, el número de establecimientos de alojamiento temporal y de alimentos y bebidas, así como el índice de vulnerabilidad al cambio climático para cada uno de los dieciocho municipios de la zona. Posteriormente, se calcularon los índices de correlación Pearson entre las variables mencionadas, encontrándose relaciones estadísticamente significativas entre la oferta turística y la vulnerabilidad climática.

Palabras clave: ecoturismo, patrimonio turístico, huasteca potosina, vulnerabilidad climática.

\begin{abstract}
The object of this research was to identify and estimate the statistical relationship that exists between the ecotourism potential, the economic activities linked to tourism such as the lodging and food and beverages services, and vulnerability to climate change, in the municipalities of the Huasteca Potosina area. Research data were obtained from the natural and cultural tourism index by municipality, the number of temporary lodging and food and beverages providers, as well as the vulnerability index to climate change for each of the eighteen municipalities in the area. Subsequently, the Pearson correlation indexes were calculated among


the aforementioned variables, finding statistically significant relationships between the tourism offering and climate vulnerability.

Keywords: ecotourism, tourism heritage, huasteca potosina, climate vulnerability.

\section{Introducción}

El turismo representa una de las actividades productivas más relevantes para la economía global, y al igual que otros sectores, se encuentra expuesto a los cambios derivados de los procesos cíclicos de la economía y a los cambios en el clima. Por lo anterior, es común que los productos turísticos sigan procesos de maduración y declive que abran el espacio para que aparezcan alternativas innovadoras acordes a nuevas necesidades, restricciones legales y ambientales y tendencias del mercado. El ecoturismo es un área de especialización que permite la generación de productos turísticos apropiados a un entorno global, donde el cuidado de los recursos naturales y la gestión ambiental juegan un papel cada vez más importante. Además, puede integrarse a las estrategias de adaptación planificada para el cambio climático.

\section{Revisión de la literatura}

El ecoturismo comprende las actividades de visita a las áreas naturales poco alteradas, con la finalidad de contemplar y estudiar su paisaje y recursos naturales y culturales (Ceballos, 1998). También, el ecoturismo se orienta hacia el desarrollo sustentable y permite la conservación de áreas de importancia cultural o biológica, generando beneficios para la población local (Carranza, 1998). Por lo tanto, existe una tendencia en los mercados internacionales a preferir destinos capaces de combinar la conservación de su patrimonio natural con un alto nivel de calidad en los servicios que se ofrecen (Torruco et al., 2013).

En ese contexto, México posee una diversidad natural impresionante comparado con otros países, al grado de ser considerado un territorio megadiverso. Inclusive, Estados como San Luis Potosí disponen de una variedad de climas y paisajes que, mediante una adecuada articulación, son idóneos para la creación de productos ecoturísticos. No obstante, poco se aprovecha este segmento. Según lo señalado por Alcántara (2014), en México se aprovecha menos del 50\% del potencial ecoturístico, que en Europa y Estados Unidos representan una derrama de aproximadamente 25000 millones de dólares al año. Otra de las características de este segmento en México, es que se encuentra poco articulado y con frecuencia se confunde con el turismo de aventura.

Cabe señalar que hoy en día ya no son los recursos naturales la única fuente de atractivos turísticos, pues los recursos culturales tangibles e intangibles han pasado a ocupar un lugar importante en la conformación de los productos turísticos; además, la incorporación de las comunidades rurales como actores protagónicos de una nueva oferta turística ha permitido la aparición de variantes tales como el ecoturismo, el etnoturismo, el 
turismo rural, el turismo comunitario y el agroturismo (Rivera et al., 2012).

En este sentido, Magio et al. (2017) consideran que el ecoturismo desarrolla las capacidades económicas de las comunidades, y ayuda a involucrar a sus habitantes en proyectos de conservación; además, puntualizan que los incentivos económicos no son los únicos motivadores de los esfuerzos conservacionistas de las comunidades.

\section{Características del estado de San Luis Potosí relacionadas con el potencial ecoturístico}

La situación geográfica del estado de San Luis Potosí, ubicado en la región centro-norte de México, le permite contar con una gran biodiversidad cuyo espectro incluye desde flora y fauna propias del semidesierto y desierto de Norteamérica (región altiplano), hasta selvas perennifolias (zona huasteca), con un importante número de especies animales y vegetales endémicas y migrantes (Rivera et al, 2012). A lo anterior se añade una tradición cultural que combina elementos prehispánicos, coloniales y modernos; en la primera categoría se inscriben tanto espacios arqueológicos como tradiciones ancestrales que sobreviven en las comunidades indígenas y mestizas asentadas hoy en día en las diversas regiones del Estado. En la segunda, una historia de siglos de explotación minera que dio origen a centros de población emblemáticos como Real de Catorce, Cerro de San Pedro, Guadalcázar o la propia ciudad de San Luis Potosí, que además de su valor histórico se ha convertido en un importante centro industrial y de negocios.

Tradicionalmente se ha regionalizado al estado de San Luis Potosí en cuatro zonas: Altiplano, Centro, Media y Huasteca. Los criterios de regionalización utilizados originalmente, parecen haber sido elementos de carácter natural, histórico y funcional (Vázquez, 2011). Entre los factores naturales, es evidente la importancia del clima; para ejemplificarlo, podemos mencionar que en los municipios del altiplano, como es el caso de Santo Domingo, predomina el clima seco templado, con una temperatura media anual de $17.5^{\circ} \mathrm{C}$, y una precipitación pluvial anual es de 474 mm (Instituto Nacional para el Federalismo y el Desarrollo Municipal [INAFED], 2018); en contraste, municipios de la huasteca, como es el caso de Xilitla, cuentan con un clima semicálido, húmedo con lluvias todo el año. La precipitación anual es de $2075.3 \mathrm{~mm}$ y la temperatura media anual es de $22^{\circ} \mathrm{C}$. 


\section{Figura 1}

\section{Microregiones de San Luis Potosi}

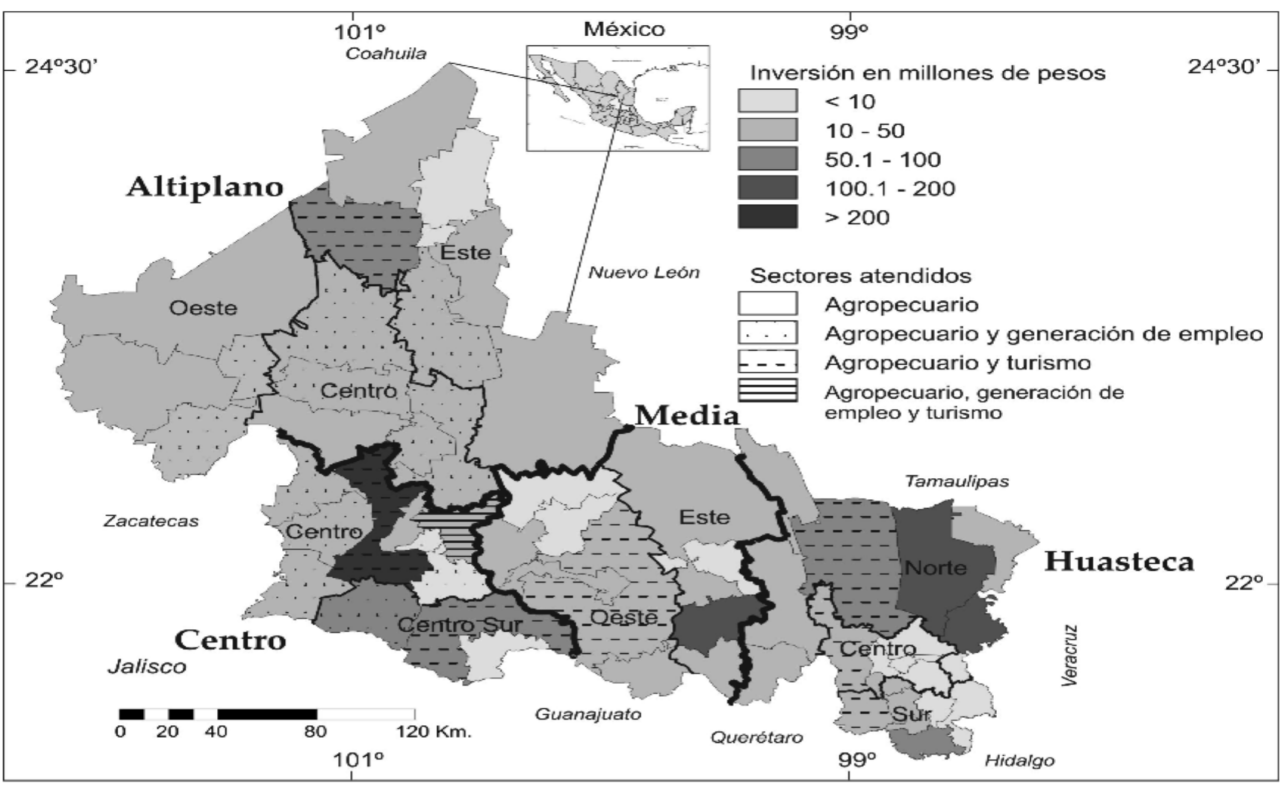

Nota. Vázquez, 2011.

Sin embargo, existen otros criterios relacionados con la vocación productiva y la demografía que gradualmente han llevado a una regionalización más precisa. Entre estos criterios se encuentran los centros de intercambio económico, el perfil productivo, y la red de vías de comunicación (Vázquez, 2011). Esta subdivisión ha dado origen a una microregionalización en la que se consideran 10 demarcaciones, de las cuales tres corresponden a la región que se presentan en este estudio: huasteca norte, huasteca centro y huasteca sur.

Como se verá más adelante, esta microregionalización juega un papel relevante, toda vez que entre las variables concurrentes para diseñar la oferta turística se encuentra la accesibilidad y el tiempo de traslado entre el lugar de alojamiento y el punto donde se encuentra el sitio de interés, así como la disposición de los actores económicos que participan en la oferta turística.

Con respecto a las características que hacen a la huasteca potosina una región especialmente atractiva se encuentran las siguientes:

Esta región se subdivide en tres subregiones fisiográficas: sierra plegada (estratos plegados en forma de mesetas, valles y lomeríos con cumbres que llegan a superar los $1000 \mathrm{~m} \mathrm{s.} \mathrm{n.} \mathrm{m.);} \mathrm{carso} \mathrm{huasteco} \mathrm{(sierras} \mathrm{y} \mathrm{cañones} \mathrm{donde}$ predominan las rocas calcáreas solubles en agua, lo que ha facilitado la formación de grutas y sótanos); y llanuras y lomeríos (donde predomina el relieve con poca inclinación). La combinación de estos tres tipos de terreno con un clima mayormente lluvioso ha dado lugar a la formación de paisajes espectaculares donde pueden encontrarse numerosos nacimientos de agua, cascadas, grutas, lagunas, y una gran diversidad biológica (Castañeda, 2010, como se 
citó en Reyes et al., 2012). En la región se registran nueve tipos de climas que pertenecen a la categoría de cálidos, semicálidos y templados, con precipitaciones que pueden alcanzar los $2500 \mathrm{~mm}$ (Reyes et al., 2012).

Suárez et al. (2020), identificaron en la zona al menos 33 geositios entre los que se encuentran cuevas de origen cárstico de donde surgen manantiales de aguas termales y sulfurosas; afloramiento de calizas y fósiles del período cretácico; límites geológicos de formaciones; caídas de agua escalonadas, cañones, vistas panorámicas, y tiros verticales de hasta $478 \mathrm{~m}$ de profundidad que resguardan ecosistemas únicos.

Sin embargo, a pesar de la abundancia de recursos naturales y culturales, subsisten problemáticas que interfieren en el adecuado aprovechamiento del potencial ecoturístico, especialmente cuando los recursos se localizan en tierras comunales (Rivera et al., 2012). Por ejemplo, los conflictos por la legítima propiedad; la falta de acuerdos sobre cómo disponer del dinero que se obtiene de los visitantes; la falta de información y capacitación de los miembros de las comunidades y los prestadores de servicios turísticos; así como la ausencia de planes de manejo integral de los sitios que definan estrategias de desarrollo local.

\section{La valoración del patrimonio turístico}

Contar con una valoración del patrimonio turístico es fundamental para desarrollar proyectos de desarrollo en el sector. Existen diferentes criterios que pueden emplearse para clasificar los recursos turísticos. Autores como Leno (1993), López (1998), Reyes y Sánchez (2005), Reyes (2006), Vázquez et al. (2010) y Reyes et al. (2012) proporcionan un marco metodológico para la valoración del acervo turístico que consiste en las siguientes tareas:

- Elaboración de un inventario de los atractivos de tipo natural y cultural de la región. Los atractivos naturales se clasifican en geológicos, geomorfológicos y áreas especiales, mientras que los culturales se clasifican en arqueológicos, arquitectónicos, así como tradiciones, fiestas y celebraciones de las comunidades. Para efectos del presente trabajo, se tomo como referencia el inventario generado por Reyes et al. (2012).

- Establecimiento de una relación cuantitativa entre el patrimonio turístico y la oferta de servicios al visitante.

- Establecimiento de una jerarquización conforme a criterios que permitan estimar su nivel de relevancia. Esta jerarquización puede indicar el valor relativo dentro de una categoría en función de factores como la singularidad, el interés artístico o la espectacularidad (Leno, 1993).

En la Tabla 1 se muestran los resultados de un estudio realizado para estimar la importancia del patrimonio turístico en la región huasteca, tomando como punto de partida la formulación de un inventario de recursos y la estimación de un índice de patrimonio turístico. (Reyes et al., 2012). En el estudio se puede apreciar que existen municipios como Aquismón y Tamasopo que destacan en patrimonio natural, mientras que otros como Ciudad Valles, Matlapa, Tampamolón Corona, Tamazunchale y Tamuín lo hacen en materia de patrimonio cultural. 


\section{Tabla 1}

Índice de patrimonio turístico de los municipios de la región Huasteca Potosina

\begin{tabular}{|l|r|r|}
\hline & \multicolumn{2}{|c|}{ Índice Turístico } \\
\hline Municipio & Natural & \multicolumn{1}{c|}{ Cultural } \\
\hline Aquismón & 40.6 & 38.6 \\
\hline Matlapa & 4.2 & 67.1 \\
\hline Tancanhuitz & 12.6 & 41.5 \\
\hline Ciudad Valles & 22.4 & 95.4 \\
\hline Coxcatlán & 0 & 25.4 \\
\hline Ébano & 18.2 & 23 \\
\hline Huehuetlán & 16.8 & 35.7 \\
\hline San Antonio & 0 & 37.2 \\
\hline San Martín Chalchicuautla & 5.6 & 41.3 \\
\hline San Vicente Tancuayalab & 0 & 48.6 \\
\hline Tamasopo & 28.8 & 34.3 \\
\hline Tamazunchale & 5.6 & 76.1 \\
\hline Tampacán & 0 & 25.6 \\
\hline Tampamolón Corona & 0 & 55.7 \\
\hline Tamuín & 8.4 & 42.2 \\
\hline Tanlajás & 15.4 & 22.6 \\
\hline Tanquián de Escobedo & 14 & 24.4 \\
\hline Axtla de Terrazas & 8.4 & 15.7 \\
\hline Xilitla & 6.2 & 51 \\
\hline El Naranjo & 11.2 & 9 \\
\hline Nota. Reys & & \\
\hline
\end{tabular}

Nota. Reyes et al., 2012.

\section{Vulnerabilidad al cambio climático y turismo}

La noción de cambio climático alude al proceso de calentamiento global que se traduce en aumento de los niveles promedio de temperatura del aire y el océano, la pérdida de hielo en los polos y zonas montañosas y el incremento en el nivel medio del mar (Grupo Intergubernamental de Expertos sobre el Cambio Climático [IPCC], 2007). Con independencia de los efectos globales previstos a largo plazo, se presentan riesgos de corto y mediano plazo a escala local y regional que pueden ser comprendidos y valorados mediante el concepto de vulnerabilidad climática.

Existen múltiples definiciones de vulnerabilidad, y por lo tanto, distintas métricas. El IPCC (2007, p. 89) la define: "Grado de susceptibilidad o de incapacidad de un sistema para afrontar los efectos adversos del cambio 
climático y, en particular, la variabilidad del clima y los fenómenos extremos". Ruiz, (2012), al revisar el marco metodológico para la definición y medición de este concepto, encuentra que si la vulnerabilidad es abordada como proceso de pérdida, implica que existe una situación ante la cual hay una transformación así como una evaluación de dicha transformación respecto a un parámetro de lo "normal" o "positivo".

El IPCC (2007, como se citó en Monterroso et al., 2013, p. 15) proporciona un marco de referencia para adaptar el concepto de vulnerabilidad a los procesos ambientales, y la define como "una función de la exposición, la sensibilidad y la capacidad adaptativa". También explica que la adaptación puede disminuir la sensibilidad al cambio climático, en tanto que la mitigación contribuye a disminuir la exposición a este fenómeno.

\section{Figura 2}

Dimensiones de la vulnerabilidad al cambio climático

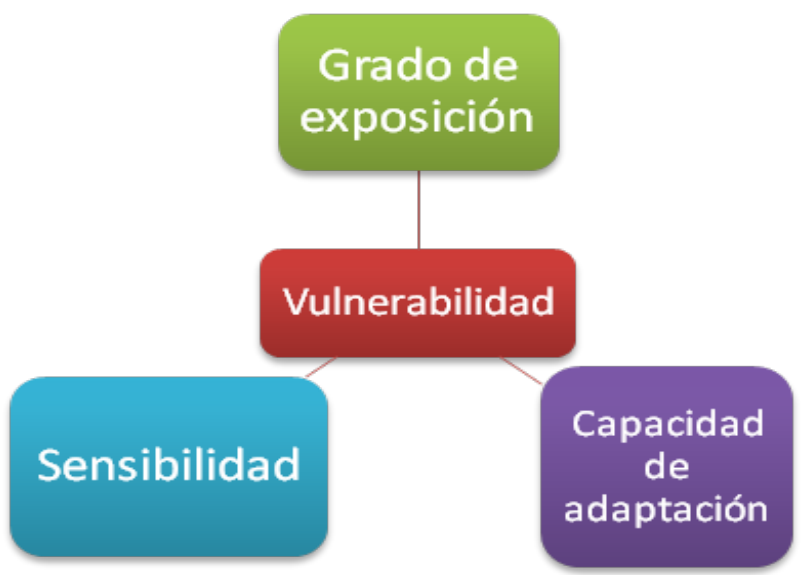

Nota. IPCC, 2007.

Basándose en la identificación de las dimensiones de la vulnerabilidad, el IPCC propone la siguiente fórmula para obtener su valor a nivel municipio:

\section{Vulnerabilidad $=\mathbf{f}[($ exposición + sensibilidad $)-$ capacidad de adaptación $]$}

El concepto de exposición se define como "el grado de estrés climático sobre una unidad particular de análisis" (Monterroso et at., 2013, p. 16). Esto puede significar cambios en las condiciones o en la variabilidad climática, o dicho de otra forma, en la rapidez y magnitud del cambio climático. Esta variable incorpora trece indicadores agrupados en las dimensiones frecuencia de eventos externos, problemática ambiental y clima y cambio climático.

La sensibilidad se interpreta como "el grado en el que un sistema resulta afectado positiva o negativamente por la variabilidad o el cambio climático" (IPCC, 2007, p. 87), con efectos directos o indirectos. Esta variable tiene una naturaleza eminentemente social y económica, y considera nueve indicadores agrupados en las dimensiones población, salud y capacidad productiva. 
Finalmente, la capacidad adaptativa refleja la "capacidad de un sistema para enfrentar los efectos del cambio climático, el potencial de implementar medidas que ayuden a disminuir los posibles impactos identificados (Monterroso et al, 2013, p. 25). En esta variable se agrupan los recursos y las capacidades tangibles e intangibles de las comunidades, que se agrupan en las dimensiones capital humano, capital social, capital financiero y capital natural.

Para efectos del presente análisis, se extrajeron del Programa Estatal de Acción ante el Cambio Climático del Estado de San Luis Potosí, los datos correspondientes a vulnerabilidad para los 18 municipios de la zona huasteca potosina.

\section{Tabla 2}

Vulnerabilidad al cambio climático en los municipios de la zona Huasteca Potosina

\begin{tabular}{lc}
\hline Municipio & Índice de vulnerabilidad \\
\hline Aquismón & 1.91 \\
Matlapa & 1.26 \\
Tancanhuitz & 1.14 \\
Ciudad Valles & -0.79 \\
Coxcatlán & 1.11 \\
Ébano & -0.32 \\
Huehuetlán & 0.79 \\
San Antonio & 1.69 \\
San Martín Chalchicuautla & 1.42 \\
San Vicente Tancuayalab & 0.311 \\
Tamasopo & 0.05 \\
Tamazunchale & 1.07 \\
Tampacán & 1.19 \\
Tampamolón Corona & 1.27 \\
Tamuín & -0.45 \\
Tanlajás & 1.46 \\
Tanquián de Escobedo & -0.07 \\
Axtla de Terrazas & 0.67 \\
Xilitla & 0.94 \\
El Naranjo & -0.89 \\
\hline
\end{tabular}

Nota. Secretaria de Ecología y Gestión Ambiental (SEGAM), 2018. 
García (2019), considera que el grado de vulnerabilidad en la zona huasteca potosina, se encuentra condicionado, tanto por las características particulares de cada municipio, como por el número y tipo de sitios turísticos, y recomienda prestar atención especial en los lugares donde se ha observado mayor variabilidad en los indicadores de precipitación y temperatura, pues esto impacta el nivel de atractivo y satisfacción que representa para los turistas, lo que a largo plazo puede ocasionar cambios en la demanda y afectación económica a las comunidades.

Como se verá más adelante, el cálculo de la vulnerabilidad climática, en su dimensión capacidad adaptativa, incluye aspectos relacionados con la formación del capital humano, social y productivo, aspectos sobre lo cuales el ecoturismo puede producir efectos positivos, teniendo en cuenta que una gestión integral de recursos puede facilitar la reconversión productiva de los municipios hacia actividades con menor impacto ambiental que las hasta ahora predominantes en la región, como es el caso de la ganadería. De ahí que el IPCC (2007) sugiere incorporar al turismo, en las estrategias de adaptación planificada, al cambio climático mediante la diversificación de las atracciones e ingresos turísticos, la planificación integrada y los incentivos financieros, aunque reconoce que existen limitaciones, ya que también podrían generarse efectos adversos sobre otros sectores, como sería el caso de la protección de especies amenazadas.

\section{Metodología}

El estudio de tipo cuantitativo, de alcance correlacional y diseño transversal. La pregunta de investigación que se plantea es la siguiente: ¿En qué grado la vulnerabilidad climática de un municipio puede ser afectada por las actividades económicas vinculadas al ecoturismo, como es la oferta de servicios de alojamiento y la oferta de servicios de alimentos y bebidas; o bien por las variables asociadas al potencial turístico, como el índice turístico natural y el índice turístico cultural?

Las variables que se utilizaron en el modelo fueron índice turístico natural, índice turístico cultural, oferta de servicios de alojamiento temporal, establecimientos de alimentos y bebidas, vulnerabilidad al cambio climático, capacidad adaptativa, sensibilidad climática y al cambio climático, y exposición climática y al cambio climático. Estas variables fueron correlacionadas entre sí, utilizando el coeficiente de correlación Pearson, para tratar de determinar la existencia de relaciones estadísticamente significativas entre las mismas. En la Tabla 3 se muestra la operacionalización de las variables. 


\section{Tabla 3}

Operacionalización de variables

\begin{tabular}{|c|c|}
\hline Variable & Forma de cálculo \\
\hline $\begin{array}{l}\text { Índice turístico natural } \\
\text { (ITN) }\end{array}$ & $\begin{array}{l}\text { Itn= } \sum[\mathrm{nj}(\mathrm{p}+\mathrm{k})] \\
\text { Donde laet = Índice de atracción ecoturística; } \mathrm{n}=\text { elemento del patrimonio turístico natu- } \\
\text { ral; } \mathrm{j}=\text { jerarquía del atractivo turístico; } \mathrm{p}=\text { factor de ponderación; } \mathrm{k}=\text { constante = } 1\end{array}$ \\
\hline $\begin{array}{l}\text { Índice turístico cultural } \\
\text { (ITC) }\end{array}$ & $\begin{array}{l}\text { Itc }=\Sigma[\mathrm{cj}(\mathrm{p}+\mathrm{k})] \\
\text { Donde Itc = Índice turístico cultural; c = elemento del patrimonio turístico cultural; j = } \\
\text { jerarquía del atractivo turístico; } \mathrm{p}=\text { factor de ponderación; } \mathrm{k}=\text { constante = } 1\end{array}$ \\
\hline $\begin{array}{l}\text { Índice de Vulnerabilidad } \\
\text { Climática (IVC) }\end{array}$ & Vulnerabilidad $=\mathrm{f}$ [(exposición + sensibilidad $)$ - capacidad de adaptación $]$ \\
\hline $\begin{array}{l}\text { Oferta de servicios de } \\
\text { alojamiento temporal }\end{array}$ & $\begin{array}{l}\text { Número de unidades económicas registradas como servicios de alojamiento temporal en } \\
\text { el Directorio Nacional de Unidades Económicas (DENUE) de INEGI. }\end{array}$ \\
\hline $\begin{array}{l}\text { Oferta de servicios de } \\
\text { alimentos y bebidas }\end{array}$ & $\begin{array}{l}\text { Número de unidades económicas registradas como servicios de alimentos y bebidas en el } \\
\text { Directorio Nacional de Unidades Económicas (DENUE) de INEGI. }\end{array}$ \\
\hline
\end{tabular}

Nota. Adaptado de Reyes et al, 2012; Monterroso, 2013; INEGI, 2018.

\section{Tabla 4}

Índice turístico natural y cultural; oferta de servicios turísticos y vulnerabilidad al cambio climático en los municipios de la zona Huasteca Potosina

\begin{tabular}{|c|c|c|c|c|c|}
\hline Municipio & $\begin{array}{l}\text { Índice } \\
\text { turístico } \\
\text { natural }\end{array}$ & $\begin{array}{l}\text { Índice } \\
\text { turístico } \\
\text { cultural }\end{array}$ & $\begin{array}{l}\text { Oferta de servicios de aloja- } \\
\text { miento temporal (número de } \\
\text { establecimientos) }\end{array}$ & $\begin{array}{l}\text { Oferta de servicios } \\
\text { de alimentos y } \\
\text { bebidas }\end{array}$ & $\begin{array}{l}\text { Índice de } \\
\text { vulnerabilidad } \\
\text { climática }\end{array}$ \\
\hline Aquismón & 40.6 & 38.6 & 3 & 36 & 1.91 \\
\hline Matlapa & 4.2 & 67.1 & 5 & 83 & 1.26 \\
\hline Tancanhuitz & 12.6 & 41.5 & 3 & 39 & 1.14 \\
\hline Ciudad Valles & 22.4 & 95.4 & 44 & 1151 & -0.79 \\
\hline Coxcatlán & 0 & 25.4 & 3 & 32 & 1.11 \\
\hline Ébano & 18.2 & 23 & 6 & 236 & -0.32 \\
\hline Huehuetlán & 16.8 & 35.7 & 3 & 9 & 0.79 \\
\hline San Antonio & 0 & 37.2 & 0 & 6 & 1.69 \\
\hline San Martín Chalchicuautla & 5.6 & 41.3 & 0 & 9 & 1.42 \\
\hline San Vicente Tancuayalab & 0 & 48.6 & 2 & 58 & 0.311 \\
\hline Tamasopo & 28.5 & 34.3 & 19 & 117 & 0.05 \\
\hline
\end{tabular}




\begin{tabular}{lccccc} 
Tamazunchale & 5.6 & 76.1 & 14 & 368 & 1.07 \\
Tampacán & 0 & 25.6 & 1 & 24 & 1.19 \\
Tampamolón Corona & 0 & 55.7 & 1 & 34 & 1.27 \\
Tamuín & 8.4 & 42.2 & 8 & 207 & -0.45 \\
Tanlajás & 1.4 & 22.6 & 0 & 36 & 1.46 \\
Tanquián de Escobedo & 14 & 24.4 & 8 & 99 & -0.07 \\
Axtla de Terrazas & 8.4 & 15.7 & 6 & 114 & 0.67 \\
Xilitla & 6.2 & 51 & 13 & 100 & 0.94 \\
El Naranjo & 11.2 & 9 & 8 & 137 & -0.89 \\
\hline
\end{tabular}

Nota. Reyes et al, 2012; Instituto Nacional de Estadística y Geografía (INEGI), 2018; SEGAM, 2018.

\section{Resultados}

Mediante la matriz de correlaciones de Pearson, se determinó el grado de dependencia lineal entre dos variables cuantitativas continuas. En este caso, el índice de vulnerabilidad climática se correlacionó con los índices de patrimonio natural y cultural, y con la oferta de servicios turísticos. En la Tabla 6 se muestran los valores $\mathrm{p}$, la significación estadística de las correlaciones que se determinaron en la Tabla 5. Los valores en negrita son diferentes a 0 con un nivel de significación alfa $=0.05$, es decir, son estadísticamente significativos.

\section{Tabla 5}

Matriz de correlaciones Pearson

\begin{tabular}{|c|c|c|c|c|c|}
\hline Variables & $\begin{array}{l}\text { Índice } \\
\text { turístico } \\
\text { natural }\end{array}$ & $\begin{array}{l}\text { Índice } \\
\text { turístico } \\
\text { cultural }\end{array}$ & $\begin{array}{l}\text { Oferta de servicios } \\
\text { de alojamiento tem- } \\
\text { poral (número de } \\
\text { establecimientos) } \\
\end{array}$ & $\begin{array}{c}\text { Oferta de } \\
\text { servicios de } \\
\text { alimentos y } \\
\text { bebidas } \\
\end{array}$ & $\begin{array}{l}\text { Índice de } \\
\text { vulnerabilidad } \\
\text { climática }\end{array}$ \\
\hline Índice turístico natural & 1 & 0.039 & 0.409 & 0.282 & -0.229 \\
\hline Índice turístico cultural & 0.039 & 1 & 0.597 & 0.647 & 0.016 \\
\hline $\begin{array}{l}\text { Oferta de servicios de aloja- } \\
\text { miento temporal (número de } \\
\text { establecimientos) }\end{array}$ & 0.409 & 0.597 & 1 & 0.923 & -0.582 \\
\hline $\begin{array}{l}\text { Oferta de servicios de alimentos y } \\
\text { bebidas }\end{array}$ & 0.282 & 0.647 & 0.923 & 1 & -0.547 \\
\hline Índice de vulnerabilidad climática & -0.229 & 0.016 & -0.582 & -0.547 & 1 \\
\hline
\end{tabular}

Nota. Elaboración propia con XLSTAT, 2016 
Tabla 6

Matriz de valores $p$

\begin{tabular}{|c|c|c|c|c|c|}
\hline Variables & $\begin{array}{l}\text { Índice } \\
\text { turístico } \\
\text { natural }\end{array}$ & $\begin{array}{l}\text { Índice } \\
\text { turístico } \\
\text { cultural }\end{array}$ & $\begin{array}{l}\text { Oferta de servicios } \\
\text { de alojamiento tem- } \\
\text { poral (Número de } \\
\text { establecimientos) }\end{array}$ & $\begin{array}{c}\text { Oferta de servicios } \\
\text { de alimentos y } \\
\text { bebidas }\end{array}$ & $\begin{array}{c}\text { Índice de } \\
\text { vulnerabilidad }\end{array}$ \\
\hline Índice turístico natural & 0 & 0.871 & 0.073 & 0.229 & 0.468 \\
\hline Índice turístico cultural & 0.871 & 0 & 0.005 & 0.002 & 0.975 \\
\hline $\begin{array}{l}\text { Oferta de servicios de } \\
\text { alojamiento temporal } \\
\text { (Número de estableci- } \\
\text { mientos) }\end{array}$ & 0.073 & 0.005 & 0 & $<0.0001$ & 0.035 \\
\hline $\begin{array}{l}\text { Oferta de servicios de } \\
\text { alimentos y bebidas }\end{array}$ & 0.229 & 0.002 & $<0.0001$ & 0 & 0.032 \\
\hline Índice de vulnerabilidad & 0.468 & 0.975 & 0.035 & 0.032 & 0 \\
\hline
\end{tabular}

Nota. Elaboración propia con XLSTAT, 2016.

Como puede apreciarse en la Tabla 5, la vulnerabilidad al cambio climático parece tener una correlación de significativa de signo negativo con actividades económicas vinculadas al turismo, como la oferta de servicios de alojamiento temporal y la oferta de servicios de alimentos y bebidas. También, se aprecian correlaciones significativas entre la oferta de servicios turísticos y el índice turístico cultural; no ocurre lo mismo con el índice turístico natural y la oferta de servicios turísticos, donde la correlación no es significativa.

Como siguiente paso en el análisis, se jerarquizaron los valores de los indicadores de la Tabla 3 en cinco categorías, conforme a los criterios que se mencionan en la Tabla 7.

Tabla 7

Ponderación cualitativa y cuantitativa de variables

\begin{tabular}{lcccccc}
\hline Jerarquías cualitativas & Valor & ITN & ITC & Hoteles & $\begin{array}{c}\text { Servicios de alimentos } \\
\text { y bebidas }\end{array}$ & IVC \\
\hline Muy bajo & 1 & 0 & $<26.0$ & $<5$ & $<50$ & $-2.0 \_$a -1.0 \\
Bajo & 2 & $3.0-15.9$ & $26.0-49.9$ & $5.0-9.0$ & $50-99$ & -0.9 a 0.0 \\
Medio & 3 & $16.0-22.9$ & $50.9-99.9$ & $10.0-14.0$ & $100-149$ & 0.1 a 1.0 \\
Alto & 4 & $23.0-38.9$ & $100.0-208.0$ & $15.0-19.0$ & $150-199$ & 1.1 a 1.5 \\
Muy alto & 5 & $>39$ & $>208.0$ & $>19$ & $>200$ & 1.6 a_2.0 \\
\hline
\end{tabular}

Nota. Adaptado de Reyes, 2012. 
Posteriormente, se obtuvo la variable nivel de atractivo turístico (NAT) al promediar los valores jerarquizados ITN e ITC de cada municipio; y oferta de servicios turísticos (OST), al promediar los valores jerarquizados de hoteles y establecimientos de alimentos y bebidas, como se muestra en la Tabla 8.

\section{Tabla 8}

Valores ponderados de las variables índice de vulnerabilidad climática (IVC), oferta de servicios turísticos (OST), y nivel de atractivo turístico (NAT)

\begin{tabular}{lcccccc}
\hline Jerarquías cualitativas & Valor & ITN & ITC & Hoteles & $\begin{array}{c}\text { Servicios de alimentos } \\
\text { y bebidas }\end{array}$ & IVC \\
\hline Muy bajo & 1 & 0 & $<26.0$ & $<5$ & $<50$ & $-2.0 \_$a -1.0 \\
Bajo & 2 & $3.0-15.9$ & $26.0-49.9$ & $5.0-9.0$ & $50-99$ & -0.9 a 0.0 \\
Medio & 3 & $16.0-22.9$ & $50.9-99.9$ & $10.0-14.0$ & $100-149$ & 0.1 a 1.0 \\
Alto & 4 & $23.0-38.9$ & $100.0-208.0$ & $15.0-19.0$ & $150-199$ & 1.1 a 1.5 \\
Muy alto & 5 & $>39$ & $>208.0$ & $>19$ & $>200$ & 1.6 a_2.0 \\
\hline
\end{tabular}

Nota. Elaboración propia.

Finalmente, se utilizó la herramienta Gráficas de Contorno de Minitab 18 para mostrar la forma en que se relacionan los valores ajustados de las variables OST y NAT con el Índice de Vulnerabilidad Climática. En la Figura 3 se aprecian las bandas de contorno de color, que representan los valores de respuesta ajustada, el agrupamiento de los municipios en al menos tres diferentes tipos de territorio; asimismo, los contornos curvos denotan la presencia de términos cuadráticos estadísticamente significativos (Minitab, 2019).

\section{Figura 3}

Gráfica de contorno de las variables Nivel de Atractivo Turístico-Oferta de Servicios Turísticos-Índice de Vulnerabilidad Climática

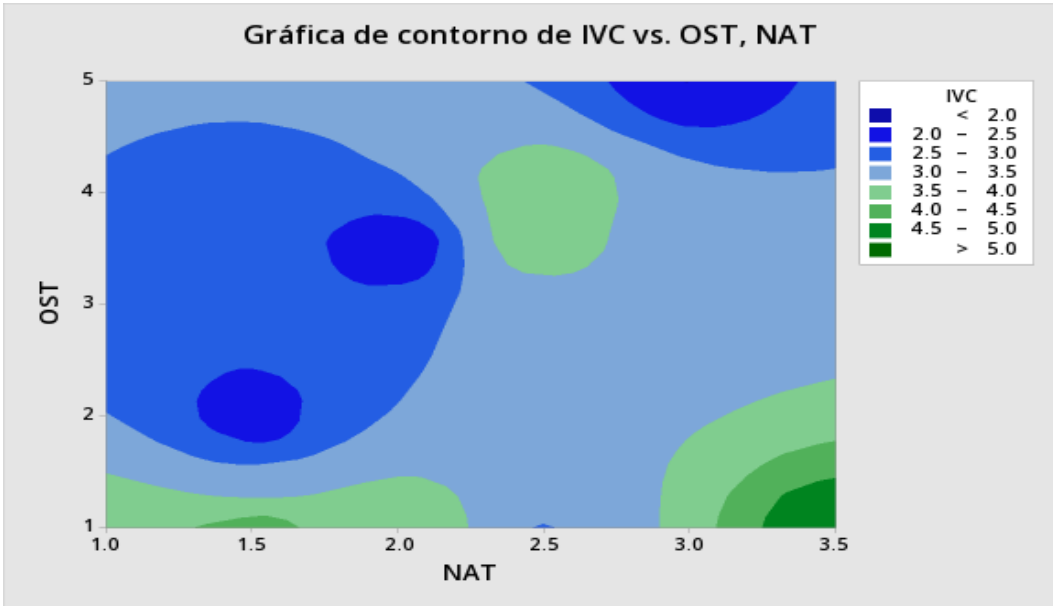

Nota. Elaboración propia con Minitab 18. 
- Territorio tipo 1. Vulnerabilidad climática alta, nivel de atractivo turístico alto, oferta de servicios turísticos baja. Este tipo de territorio sugiere la presencia de altos niveles de sensibilidad y bajos niveles de capacidad adaptativa, y por lo tanto, requiere gestionar proyectos integrales enfocados en el aprovechamiento del potencial turístico para desarrollar capacidades tangibles e intangibles de las comunidades, sin vulnerar los ecosistemas. En esta categoría se ubica el municipio de Aquismón, que en el año 2020 obtuvo el reconocimiento como Pueblo Mágico, lo que previsiblemente mejorará su capacidad de gestión.

- Territorio tipo 2. Vulnerabilidad climática media-baja, nivel de atractivo turístico alto, oferta de servicios turísticos alta. En este territorio se observa la existencia de polos de desarrollo urbano cerca de los parajes naturales y sitios culturales y arqueológicos, lo que ha permitido su aprovechamiento; requiere evaluar el efecto de las actividades turísticas sobre los ecosistemas, con el fin de prevenir su deterioro como consecuencia de las actividades humanas. Entre los municipios que conforman este tipo de territorio, destaca Ciudad Valles, que es el principal centro urbano de la huasteca potosina, y Xilitla, que es el primer municipio de la región en obtener el reconocimiento como Pueblo Mágico.

- Territorio tipo 3. Vulnerabilidad climática media-baja, nivel de atractivo turístico medio-bajo, oferta de servicios turísticos media-baja. En esta categoría se encuentran municipios cuya capacidad adaptativa se ha gestionado mediante proyectos de desarrollo agrícola y de desarrollo social; podrían aprovechar mejor su potencial sin vulnerar los ecosistemas mediante proyectos de ecoturismo y turismo rural, enfocados a segmentos de mercado bien delimitados, cuyos intereses van más allá de la visita a parajes naturales o sitios culturales reconocidos.

\section{Conclusiones}

El turismo, como cualquier actividad económica, genera impactos ambientales que en algún grado pueden incidir sobre la vulnerabilidad climática de los municipios. Sin embargo, como se apuntó al inicio del artículo, la tendencia hacia el ecoturismo privilegia la noción de desarrollo sustentable, y puede facilitar la reconversión productiva hacia actividades con menor impacto ambiental que la ganadería, la industria, la explotación forestal y algunas actividades agrícolas. Además facilita la formación de capital humano, social y financiero pues impulsa la formación de nuevas unidades económicas y la capacitación del personal para el desarrollo de nuevas tareas, como es la atención de los visitantes y la conservación del patrimonio natural y cultural.

Mediante el ejercicio de correlación de variables realizado, se encontraron relaciones significativas de signo negativo entre las actividades vinculadas al turismo, como la oferta de servicios de alojamiento temporal y de alimentos y bebidas, con la vulnerabilidad climática. Esto nos sugiere que las actividades relacionadas con el turismo pueden tener un efecto favorable sobre las dimensiones de la vulnerabilidad climática, en especial sobre la capacidad de adaptación. Cabe recordar que en esta variable se reflejan las dimensiones capital humano, social, financiero y natural, las cuales se relacionan de manera cercana con la configuración de la oferta 
turística.

Por otra parte, también se observa una baja correlación entre la oferta de servicios turísticos y los índices de atractivo turístico y cultural; sugiere que en la mayoría de los municipios existe un déficit en la oferta de servicios, ya que estos se centralizan en Ciudad Valles. El alojamiento ecológico integrado a la naturaleza puede ser una alternativa para mejorar la oferta de servicios, y especialmente para integrar a las comunidades como actores clave en el sector.

Una estrategia de integración de las comunidades en el diseño de productos ecoturísticos requiere un esfuerzo de capacitación y organización que las instituciones educativas pueden ayudar a conducir, pues se requiere integrar las dimensión técnica, financiera y de mercado, planes de manejo integral y aspectos legales, pues como se apuntó en el marco teórico, subsisten situaciones de conflicto entre comunidades por la propiedad, el usufructo y la distribución de los beneficios que producen los recursos turísticos. Un componente clave para cualquier proyecto de desarrollo integral debería ser el uso del conocimiento científico y la educación para la sustentabilidad, pues con ello se facilita la transferencia de saberes hacia las comunidades bajo un enfoque de sustentabilidad (Cujía et al., 2017).

En este sentido, es importante advertir que sin una política de desarrollo turístico sustentable, es fácil que aún en el contexto del ecoturismo se permita el saqueo de especies endémicas, amenazadas o en peligro de extinción, o bien que se enajenen las capacidades de control del territorio en perjuicio de las comunidades locales (Guzmán, 2016).

\section{Conflicto de intereses}

El autor $\square$ o tienen conflicto de intereses con el contenido de este trabajo.

\section{Referencias}

Alcántara, C. (2014, 29 de septiembre). Ecoturismo, segmento desaprovechado para atraer viajeros a México. El Financiero [Empresas]. http://www.elfinanciero.com.mx/empresas/ecoturismo-segmento-desaprovechado-para-atraer-viajeros-a-mexico

Carranza, M. (1998). Ecoturismo en México. Revista del Centro de Investigación de la Universidad La Salle, 3(11), 287.

Ceballos, H. (1998). Ecoturismo. Naturaleza y Desarrollo Sostenible. Editorial Diana.

Cujía, E., Pérez, S. y Maestre, D. (2017). Ecoturismo, educación, ciencia y tecnología, factores de desarrollo sustentable: caso La Guajira, Colombia. Educación y Humanismo, 19(32), 174-189. https://doi.org/10.17081/eduhum.19.32.2540

Guzmán, M. (2016). El impacto del turismo en la conservación de la biodiversidad en San Luis Potosí. Sociedad y Ambiente, (11), 148-159.

Instituto Nacional de Estadística y Geografía (INEGI). (2018). Directorio Nacional de Unidades Económicas (DENUE). http://www. beta.inegi.org.mx/app/mapa/denue/

Instituto Nacional para el Federalismo y el Desarrollo Municipal (INAFED). (2018). Enciclopedia de los Municipios y Delegaciones de México. http://www.inafed.gob.mx/work/enciclopedia/EMM24sanluispotosi/index.html 
García, A. (2019). Vulnerabilidad de los sitios turísticos en la región Huasteca, San Luis Potosí, como resultado de la variabilidad climática [Tesis de maestría, Universidad Autónoma de San Luis Potosí]. http://148.224.97.92/xmlui/handle/i/5730

Grupo Intergubernamental de Expertos sobre el Cambio Climático (IPCC). (2007). Cambio climático 2007: Informe de síntesis. Contribución de los grupos de trabajo I, II y III al cuarto informe de evaluación del grupo intergubernamental de expertos sobre el cambio climático [Equipo de redacción principal: Pachauri, R. K. y Reisinger, A. (directores de la publicación)]. Ginebra, Suiza.

Grupo Intergubernamental de Expertos sobre el Cambio Climático (IPCC). (2012). Summary for Policymakers. In C. B., Field, V. Barros, T. F. Stocker, D. Qin, D. J. Dokken, K. L. Ebi, M. D. Mastrandrea, K. J. Mach, G. K. Plattner, S. K. M. Allen, M. Tignor, \& P. M. Midgley (eds.), Managing the Risks of Extreme Events and Disasters to Advance Climate Change Adaptation [A Special Report of Working Groups I and II of the Intergovernmental Panel on Climate Change] (pp. 1-19). Cambridge University Press.

Leno, F. (1993). La evaluación de los recursos turísticos. El caso del Canal de Castilla (t. ii). Universidad Complutense.

López, D. (1998), La ordenación y planificación integrada de los recursos territoriales turísticos: estudio práctico de un espacio de "desarrollo turístico incipiente" el Alto Palancia (Castellón). Universitat Jaume I. Castellón.

Magio, K., Guillén, E. y Carballo, E. (2017). Ecoturismo y conservación en el ejido Ruiz Cortines, Los Tuxtlas. Teoría y Praxis, 22, 159-195. http://www.teoriaypraxis.uqroo.mx/doctos/numero22/Obombo-etal.pdf

Minitab Statistical Soffware. (2019). Soporte Minitab 18. http://bit.ly/3qx5TWH

Monterroso, R., Fernández, E., Trejo, V., Conde, A., Escandón, C., Villers, R. y Gay, G. (2013). Vulnerabilidad y adaptación a los efectos del cambio climático en México. [Centro de Ciencias de la Atmósfera - Programa de Investigación en Cambio Climático]. Universidad Nacional Autónoma de México.

Reyes, Ó. (2006). La evaluación de las condiciones naturales para el desarrollo sustentable de la actividad turística en el estado de Oaxaca [Tesis doctoral, Universidad Nacional Autónoma de México]. México.

Reyes, Ó. y Sánchez, A. (2005). Metodología para determinar el potencial de los recursos turísticos naturales en el estado de Oaxaca, México. Cuadernos de Turismo, 16, 153-173.

Reyes, Ó., Vázquez, V., Reyes, H., Caretta, M. y Rivera, J. (2012). Potencial turístico de la región Huasteca del estado de San Luis Potosí, México. Economía, sociedad y territorio, 12(38), 249-275.

Rivera, J., Vázquez, V., Reyes, H., Reyes, Ó. y Caretta, M. (2012). Potencialidades y desafíos del turismo y ecoturismo en el estado de San Luis Potosí, México: retos y expectativas para alcanzar el desarrollo regional. PASOS. Revista de Turismo y Patrimonio Cultural, 10(3).

Ruiz, N. (2012). La definición y medición de la vulnerabilidad social. Un enfoque normativo. Investigaciones geográficas, (77), 63-74. http://www.scielo.org.mx/scielo.php?script=sci_arttext\&pid=S0188-46112012000100006

Secretaría de Ecología y Gestión Ambiental (SEGAM). (2018). Programa estatal de acción ante el cambio climático de San Luis Potosí 2018. https://slp.gob.mx/segam/Documentos\%20compartidos/ESTUDIOS\%20PROGRAMAS\%20Y\%20PROYECTOS/ PEACC\%202019\%20SLP\%20V1.pdf

Suárez, I., Meraz, G., Gutiérrez, A. y Turrubiartes, H. (2020). Identificación del geopatrimonio para la construcción del Geoparque Huasteca Potosina. Tlatemoani. Revista académica de investigación, 11(34), 207-228.

Torruco, D., González, M. y Torruco, A. (2013). Las playas de Quintana Roo: sus riesgos y vulnerabilidad. El Periplo Sustentable, (24), 155-172. https://rperiplo.uaemex.mx/article/view/5000

Vázquez, V. (2011). Nuevas regiones del estado de San Luis Potosí: una expresión territorial de su integración funcional para la competitividad económica. Investigaciones geográficas, (75), 103-117.

Vázquez, V., Reyes, O., Rivera, J., Nicolás, M. y Reyes, H. (2010). Evaluación de los atractivos naturales para el desarrollo del ecoturismo en la región Huasteca de San Luis Potosí, México. Cuadernos de Turismo, 25, 229-245. 
Francisco Javier Segura Mojica

Profesor del Tecnológico Nacional de México/Instituto Tecnológico de San Luis Potosí, donde actualmente es Presidente de la Academia de Administración. Doctor en Administración Pública por la Universidad Complutense de Madrid, con Maestría en Administración de Instituciones Educativas por el Centro de Investigación para la Administración Educativa, y licenciado en Relaciones Industriales por el Instituto Tecnológico de San Luis Potosí. Como profesor investigador, cuenta con Perfil Prodep desde el año 2009 y es integrante del Cuerpo Académico "Empresas, Tecnología y Sociedad del Conocimiento y la Información"

recursosmx@yahoo.com

ORCID: http://orcid.org/0000-0001-5981-9246 